\title{
Characterization of silicon 3D pixel detectors for the ATLAS Forward Physics experiment
}

\author{
I. López Paz*, E. Cavallaro*, S. Grinstein*† and J. Lange* \\ * Institut de Física d'Altes Energies (IFAE) \\ 08193 Bellaterra (Barcelona), Spain \\ $\dagger$ ICREA
}

\begin{abstract}
The ATLAS Forward Physics (AFP) project aims to measure protons scattered under a small angle from the $p p$ collisions in ATLAS. In order to perform such measurements, a new silicon tracker, together with a time-of-flight detector for pile-up removal, are planned to be installed at $\sim 210 \mathrm{~m}$ away from the interaction point and at $2-3 \mathrm{~mm}$ away from the LHC proton beam. To cope with such configuration and maximize the physics outcome, the tracker has to fulfil three main requirements: endure highly non-uniform radiation doses, due to the very inhomogeneous beam profile, have slim and efficient edges to improve the acceptance of the tracker, and provide good position resolution.

Recent laboratory and beam test characterization results of AFP prototypes will be presented. Slim-edged 3D pixel detectors down to 100-200 $\mu \mathrm{m}$ were studied and later non-uniformly irradiated (with a peak fluence of several $10^{15} \mathbf{n}_{e q} / \mathbf{c m}^{2}$ ) to determine the fulfilment of the AFP requirements.
\end{abstract}

Index Terms-AFP, 3D pixels, radiation hardness, high energy physics.

\section{INTRODUCTION}

$\mathbf{T}$ HE planned ATLAS Forward Physics (AFP) system [1] consists of a combination of a tracker detector with a time-of-flight detector (ToF) for background pile-up removal at $\sim 210 \mathrm{~m}$ away from the interaction point of ATLAS, placed in 2 Roman Pot stations at each side of the interaction point at a distance of 2-3 $\mathrm{mm}$ from the LHC proton beam. The 4 planes of the tracker in each Roman Pot will be mounted almost perpendicular to the proton beam, under a small tilt of $15^{\circ}$. The goal of this new ATLAS sub-detector will be to measure energy loss and momentum transfer of protons in diffractive processes in which at least one of the protons leaves the interaction point intact from the $p p$ collisions in the ATLAS detector.

Given the detector configuration, the requirements for the tracker are listed below:

1) Have a good spatial resolution, i.e. $10 \mu \mathrm{m}(30 \mu \mathrm{m})$ in the horizontal (vertical) direction to maximize energy and momentum resolution,

2) have a small non-instrumented edge, with a dead area of maximally $100-200 \mu \mathrm{m}$ to maximize the acceptance and probe the lowest range of proton momentum loss, and

3) be able to cope with highly non-uniform fluences to maintain a high and uniform efficiency during operation, due to the non-uniform beam profile expected in the AFP planes.
The tracker in each of the Roman pots will consist of 4 planes of radiation hard silicon 3D pixel sensors with columnlike electrodes penetrating the substrate of the silicon. Such sensors have been developed for the ATLAS Insertable BLayer (IBL) and proved to be radiation hard to fluences of $5 \times 10^{15} \mathrm{n}_{e q} / \mathrm{cm}^{2}$ under uniform irradiation [2]. The same sensor design and electronics front-end will be used in AFP with a critical modification, slim-edging, which will be presented in the following sections.

AFP has been approved to be operated in a low pileup scenario, i.e. for dedicated runs with low luminosity in which the number of interactions per bunch crossing is less than 1. A possible later upgrade of the AFP detector for a high-luminosity (high pile-up) scenario would require the tracker to cope with a fluence peak of around $3 \times 10^{15} \mathrm{n}_{e q} / \mathrm{cm}^{2}$, orders of magnitude higher than the expected fluence in the low luminosity plan. The irradiation studies presented here are targeted at the harsh requirements of the high-luminosity scenario.

The well functioning of the AFP prototypes after slimedging has been proven and efficiency after non-uniform irradiation has been studied [3], [4]. A review of previous results with updated and more recent data will be presented: voltage dependence of the efficiency after non-uniform irradiation and studies of the edge sensitivity after irradiation obtained from recent testbeams are presented. New studies on position resolution will be shown in addition.

\section{The Slim-Edged AFP Prototypes}

The recently installed ATLAS IBL pixel detector uses the FE-I4 [5] readout chip, which has been designed to sustain $250 \mathrm{Mrad}$, the dose foreseen for the IBL lifetime. Designed in $130 \mathrm{~nm}$ technology, this front-end features $336 \times 80$ channels with $50 \times 250 \mu \mathrm{m}^{2}$ pitch each, the active area covering thus about $16.8 \times 20.0 \mathrm{~mm}^{2}$. Each channel is DC coupled to the corresponding pixel on the sensor side via bump-bonding.

IBL 3D sensors were fabricated in $230 \mu \mathrm{m}$ thick wafers with a double sided process, where $\mathrm{n}$ - and p-type columns penetrating the silicon perpendicular to the wafer's surface are etched from the opposite sides of the substrate. One pixel is formed by two n-columns surrounded by six p-columns and matches in size and pitch the FE-I4 channels.

Two vendors have been fabricating the 3D sensors for IBL with different designs: CNM (Centro Nacional de Microelectrónica, Barcelona, Spain) [6] and FBK (Fondazione 


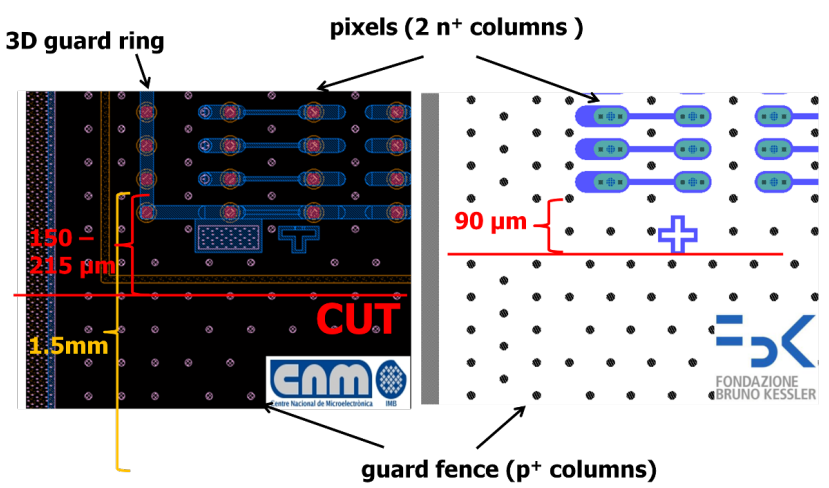

Fig. 1. CNM (left) and FBK (right) termination designs. In red are shown the cut lines for the AFP prototypes.

Bruno Kesler, Trento, Italy) [7]. The main differences between the two designs are:

- The FBK solution features passing-through electrode columns, isolated by p-spray, while in the CNM design, the columns do not traverse the full thickness of the wafer and are isolated with a p-stop on the n-column side.

- The active pixelated area is terminated in both cases with a p-column ohmic fence. However, the CNM design provides a 3D guard-ring in addition (see Fig. 1).

Both 3D IBL designs have an edge extension of $200 \mu \mathrm{m}$ along the 2 external pixel columns. However, the AFP-relevant side foreseen to face the beam (opposite to wirebond side) features a $\sim 1.5 \mathrm{~mm}$ inactive edge along the short pixel (row) direction (bottom side in Fig. 1). For the AFP prototypes, that dead region is cut down to meet the AFP acceptance requirements, leaving a remaining edge extension of $\sim 90$ $215 \mu \mathrm{m}$ (see Tab. I). The standard diamond saw technique was studied here to perform the slim-edging of IBL sensors for the prototypes, and eventually selected for the AFP detectors due to the possibility of both sensor vendors to perform it on site, saving production time and cost.

The first AFP tracker module prototypes presented in this paper are based on modules rejected from the IBL production, so not the best quality is expected.

\section{LABORATORY CHARACTERIZATION}

Leakage current versus bias voltage measurements (socalled IV curves) have been performed on the slim-edged devices with the standard diamond saw technique, which confirmed that the slimming process to $\sim 100-200 \mu \mathrm{m}$ does not introduce defects on the substrate that affects the electrical performance [4].

The obtained results are consistent with other published results where the edge of 3D FBK sensors were cut at different distances from the last readout column with no changes on electrical behaviour down to $75 \mu \mathrm{m}$ [8].

The mean noise of the AFP prototypes was measured in the laboratory. The average measured noise of the CNM devices ranges from 150 to $160 \mathrm{e}^{-}$whereas for FBK a noise of $200 \mathrm{e}^{-}$ was observed, wich is slightly higher than the IBL average of 130-140 $\mathrm{e}^{-}$[2] but such noise levels are typical of the IBLspare quality devices. Only one CNM device shows up to $40 \%$ higher noise in the pixel row closest to the slim edge, while the other prototypes exhibit a uniform noise around the last pixel rows. Regardless of the difference, a standard threshold of $2 \mathrm{ke}^{-}$ensures no impact on operation.

\section{Testbeam CAmpaigns}

Several testbeam campaigns have been carried out in order to characterize and qualify the slim-edged AFP 3D prototypes at DESY (Deutsches Elektronen-Synchrotron), which provides 4-5 GeV electrons, and at CERN SPS (Super Proton Synchrotron), which offers a $120 \mathrm{GeV}$ pion beam to the users.

In all testbeam measurements, the ACONITE telescope based on EUDET [12] has been used for track interpolation to the device under test. In scenarios where multiple scattering is low, e.g. at CERN SPS, the telescope provides $4 \pm 1 \mu \mathrm{m}$ pointing resolution whereas with the 4-5 GeV electrons at DESY the precision for the configuration in which the telescope was operated is around $15 \mu \mathrm{m}$. The following results were obtained with the devices under test under normal incidence with respect to the beam direction.

The edge efficiency after slim-edging was measured in four AFP prototypes, two FBK (FBK-S1-R9 and FBK-S2R10) and two CNM (CNM-S3-R5 and CNM-S5-R7) sensors. The respective physical edge extension after the edge cut is shown in Tab. I. For comparison, the edge efficiency was also studied in other non-slim-edged FBK devices (FBK-9, FBK11 and FBK-13), two of them (FBK-9 and FBK11) irradiated uniformly at two different fluences $\left(2\right.$ and $5 \times 10^{15} \mathrm{n}_{e q} / \mathrm{cm}^{2}$ respectively) and annealed for $120 \mathrm{~min}$ at $60^{\circ} \mathrm{C}$.

Moreover, non-uniform irradiation was performed at CERNPS (CNM-57) and at Karlsruhe with an FBK device (FBK-1202-08) and the aforementioned CNM slim-edged prototypes (i.e. CNM-S3-R5 and CNM-S5-R7).

The thresholds of all the devices-under-test ranged from 2 to $3 \mathrm{ke}^{-}$, and the Time-over-Threshold (ToT) response was calibrated to 8-11 ToT at a charge collected of $20 \mathrm{ke}^{-}$ ( $1 \mathrm{ToT}=25 \mathrm{~ns}$ ). The measurements of irradiated sensors were performed at temperatures between -15 and $-50^{\circ} \mathrm{C}$ to reduce the leakage current (dry-ice cooling).

\section{A. Edge Efficiencies}

The understanding of the charge collection at the edges of the sensor is vital in the AFP framework, in particular, the efficiency around the last instrumented row of pixels opposite the wirebonds, which will face the beam in the AFP detector (but also the other side was measured for comparison)

In Fig. 2, the projection of the efficiency around the AFPrelevant edge is shown for a CNM (biased at $30 \mathrm{~V}$ ) and an FBK (biased at 20V) sample. The behaviour of the sensitivity extension, i.e. the distance from the last pixel to the $50 \%$ point of an error function fit, is clearly different for both designs. The 3D guard-ring present in the CNM design prevents the collection of charges beyond the instrumented area, while such constraint does not exist in the FBK sensors without a guardring, where also charge deposited in the extended depleted area beyond the last pixel row is collected. 

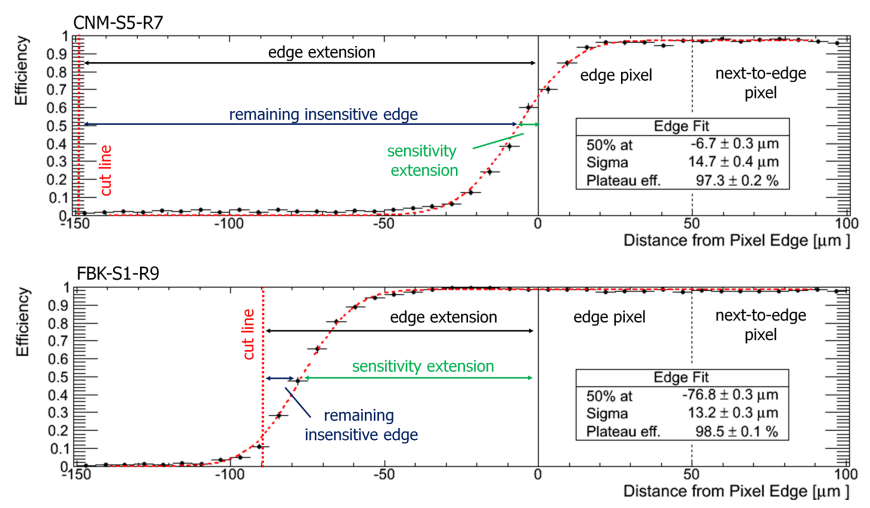

Fig. 2. Efficiency around last pixel row for an un-irradiated and slimedged CNM sensor (above) and FBK sensor (below) measured at DESY. The smearing of the curve is caused by the telescope resolution $(\sim 12-15 \mu \mathrm{m})$.

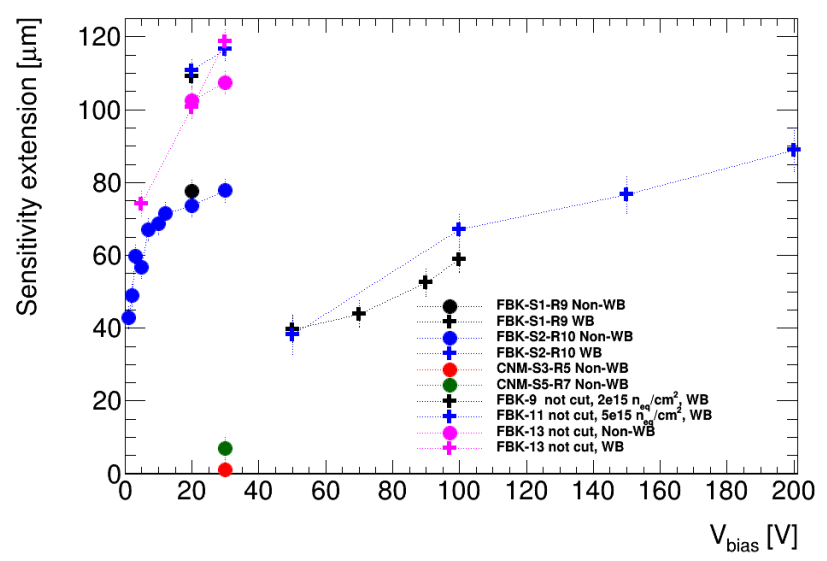

Fig. 3. Sensitivity extension beyond the last pixel as a function of voltage. Sensors FBK-9, 11 and 13 were not slim-edged. The error bars represent the uncertainties from the error function fit and $3 \mu \mathrm{m}$ of systematic uncertainties.

As can be seen in Fig. 3, there is a voltage dependence of the sensitivity extension of the FBK sensors, meaning that the charge collecting region after the last instrumented pixel grows parallel to the wafer surface with increasing voltage. At large enough voltages (around $20 \mathrm{~V}$ ), the sensitivity extension reaches a plateau.

Measurements of the uniformly irradiated and non-slimedged devices (also added ing Fig. 3) show that the sensitivity extension is still present but reduced due to radiation-induced changes in the depletion zone and trapping of the charge carriers, especially at low voltages.

The edge efficiency was also measured at the edge opposite to the slim-edged side, as well as in detectors that were not slim-edged (FBK-13): it has been observed that the sensitivity extension grows significantly larger than at the slim-edged side. This effect might be caused by cut-induced defects at the slim-edged side and is still under study. For a better understanding, simulations are needed in the future to complement the measurements.

A summary table of the devices under test and the maximum efficiency extension are shown in Tab. I, together with the distance from the edge to the last p-column forming a pixel. To conclude, CNM sensors feature a well defined active area termination while the last pixels at FBK devices can collect charge from 60-90 $\mu \mathrm{m}$ away from the last pixel row even after irradiation. This effect implies a remaining insensitive edge of only 12-14 $\mu \mathrm{m}$ in the slim-edged side of non-irradiated sensors, which is to the knowledge of the authors the slimmest inactive edge apart from fully active edge sensors [9], [10]. However, such behaviour can affect the position resolution of the device at the edge. Also the CNM sensors fulfil the edge requirements of AFP, with a remaining inactive area of 143$214 \mu \mathrm{m}$ width.

\section{B. Efficiency After Non-Uniform Irradiation}

The non-uniform fluence distribution expected in the AFP tracker may affect the hit efficiency of the system. The voltage needed to achieve maximum efficiency of the silicon sensor increases as a function of the fluence. It might occur the case that the voltage needed for full efficiency in the highestirradiated area of the sensor is larger than the breakdown voltage in the area where the fluence level is lower because the breakdown voltage usually increases with fluence. In such a scenario, a high enough voltage to efficiently operate the highly irradiated region would lead to a large increase of the leakage current of the full device, since the non-irradiated zone would be in breakdown, hence making the device nonoperable.

The expected fluence for the silicon tracker planes during AFP operation has been simulated in the past for a high luminosity scenario [11]. A maximum of $5 \times 10^{15} \mathrm{p} / \mathrm{cm}^{2}$ $\left(3 \times 10^{15} \mathrm{n}_{e q} / \mathrm{cm}^{2}\right.$ assuming the hardness factor for $\mathrm{GeV}$ protons) was observed in the simulations in a localized area of a few $\mathrm{mm}^{2}$ and a decrease of several orders of magnitude in the vicinity. The simulation does not take the background effects into account, which is expected to add a uniform fluence distribution from lower-energy particles. For a low luminosity scenario, for which AFP has been currently approved, the quoted fluence is expected to be 3 orders of magnitude smaller.

In order to test the devices under the non-uniform fluence scenario, several irradiation campaigns were carried out. The devices were irradiated at the following irradiation facilities:

- CERN-PS with a focused beam of $23 \mathrm{GeV}$ protons with a peak fluence of $4 \times 10^{15} \mathrm{n}_{e q} / \mathrm{cm}^{2}$. The fluence gradient is not as large as expected during AFP operation: an area of around $1 \mathrm{~cm}$ received a fluence larger than $10^{15} \mathrm{n}_{e q} / \mathrm{cm}^{2}$ (see Fig. 4, top left).

- KIT (Karlsruhe Institute of Technology) with a proton beam of $23 \mathrm{MeV}$, where the modules were covered by a $5 \mathrm{~mm}$ thick aluminium shield with a $3 \mathrm{~mm}$ diameter circular hole (see Fig. 4, top center) or a $4 \mathrm{~mm}$ wide slit hole (see Fig. 4, top right) to achieve a highly nonuniform profile. The target fluence achieved ranged from 2 to $3.6 \times 10^{15} \mathrm{n}_{e q} / \mathrm{cm}^{2}$.

The hit efficiency was measured in samples irradiated with these different scenarios, and the efficiency maps for the highest measured voltage are shown in Fig. 4. It can be seen how the efficiency is almost uniform after irradiation. A small degradation of the efficiency in the irradiated area of the sensor (named irradiated half and irradiated centre in 
TABLE I

EFFICIENCY EXTENSION, SENSITIVITY BEYOND THE LAST INSTRUMENTED PIXEL AND REMAINING INSENSITIVE EDGE OF THE DEVICES UNDER TEST [4]. All VALUes REFer to THE MAXimum MEASURED VOLTAGe.

\begin{tabular}{|c|c|c|c|c|c|c|c|c|c|c|}
\hline & \multicolumn{6}{|c|}{ Slim-edged AFP prototype } & \multicolumn{4}{|c|}{ Not slim-edged } \\
\hline Sample & \multicolumn{2}{|c|}{ FBK-S1-R9 } & \multicolumn{2}{|c|}{ FBK-S2-R10 } & \multirow{2}{*}{$\frac{\text { CNM-S3-R5 }}{\text { Non-wb }}$} & \multirow{2}{*}{$\begin{array}{r}\text { CNM-S5-R7 } \\
\text { Non-wb }\end{array}$} & \multirow{2}{*}{$\begin{array}{r}\text { FBK-9 } \\
\mathrm{Wb}\end{array}$} & \multirow{2}{*}{$\begin{array}{r}\text { FBK-11 } \\
\mathrm{Wb}\end{array}$} & \multicolumn{2}{|c|}{ FBK-13 } \\
\hline Edge side [Wirebond/Non-wb] & Non-wb & $\mathrm{Wb}$ & Non-wb & $\mathrm{Wb}$ & & & & & Non-wb & $\mathrm{Wb}$ \\
\hline Irradiation $\left[\mathrm{n}_{e q} / \mathrm{cm}^{2}\right]$ & \multicolumn{2}{|c|}{0} & \multicolumn{2}{|c|}{0} & 0 & 0 & $2 \times 10^{15}$ & $5 \times 10^{15}$ & \multicolumn{2}{|c|}{0} \\
\hline Maximum measured voltage & \multicolumn{2}{|c|}{$20 \mathrm{~V}$} & \multicolumn{2}{|c|}{$30 \mathrm{~V}$} & $30 \mathrm{~V}$ & $30 \mathrm{~V}$ & $100 \mathrm{~V}$ & $200 \mathrm{~V}$ & \multicolumn{2}{|c|}{$30 \mathrm{~V}$} \\
\hline Edge extension after cut & $91 \mu \mathrm{m}$ & - & $87 \mu \mathrm{m}$ & - & $215 \mu \mathrm{m}$ & $150 \mu \mathrm{m}$ & - & - & \multicolumn{2}{|c|}{-} \\
\hline Sensitivity beyond last pixel & $77 \mu \mathrm{m}$ & $107 \mu \mathrm{m}$ & $75 \mu \mathrm{m}$ & $117 \mu \mathrm{m}$ & $1 \mu \mathrm{m}$ & $7 \mu \mathrm{m}$ & $59 \mu \mathrm{m}$ & $90 \mu \mathrm{m}$ & $107 \mu \mathrm{m}$ & $119 \mu \mathrm{m}$ \\
\hline Remaining insensitive edge & $14 \mu \mathrm{m}$ & - & $12 \mu \mathrm{m}$ & - & $214 \mu \mathrm{m}$ & $143 \mu \mathrm{m}$ & - & - & \multicolumn{2}{|c|}{-} \\
\hline
\end{tabular}
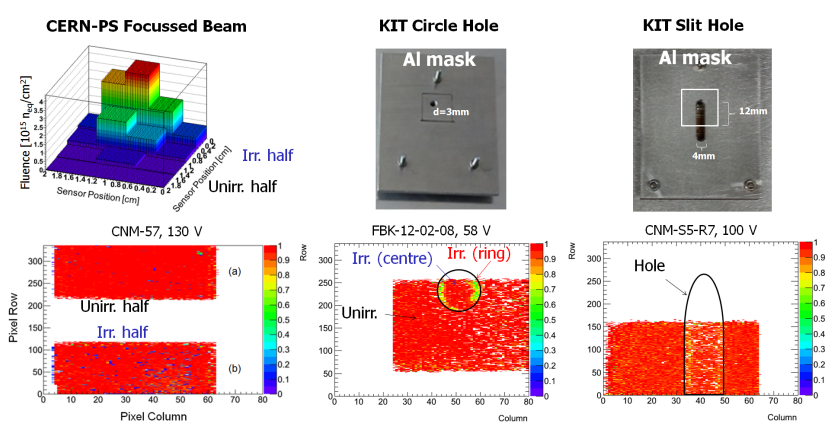

Fig. 4. Hit efficiency map measured after non-uniform irradiation at CERN PS with a focused beam (fluence profile shown above) and at KIT with an aluminium shield with a slit/hole.

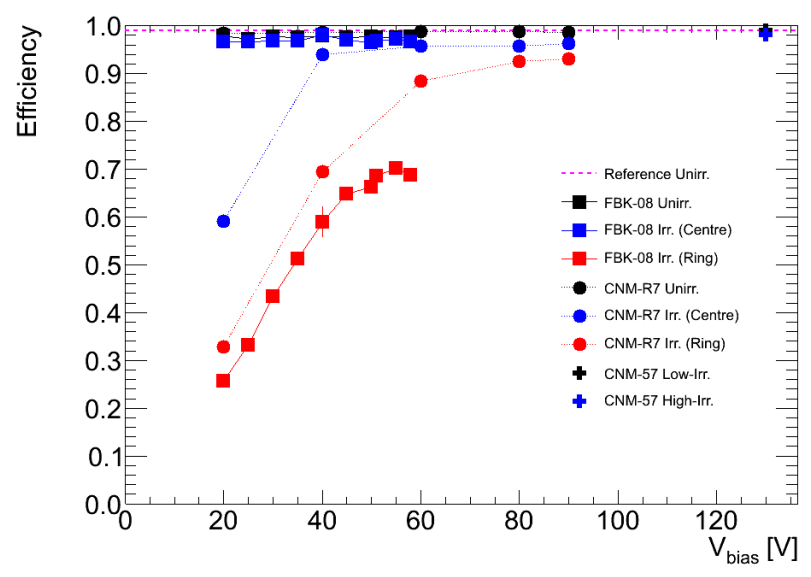

Fig. 5. Hit efficiency as a function of bias voltage for different devicesunder-test after non-uniform irradiation for different irradiation region (nonirradiated, irradiated centre and irradiated ring). The hit efficiency of a reference non-irradiated sensor is also shown as a dashed line.

the figure) is observed. At the highest measured voltage, the difference in efficiency between the non-irradiated region and the irradiated (half/centre) one is at the percent level and the efficiency is always larger than $96 \%$ at the maximum voltage applied. This difference is larger at lower voltages as can be seen in Fig. 5, where the efficiency in the non-irradiated and irradiated (half/centre) area are plotted as a function of applied bias voltage.

In the KIT irradiation efficiency map a third region (named irradiated ring in Fig. 4) can be differentiated in which the efficiency is smaller than at the centre of the hole and the non- irradiated region, which corresponds to the edge of the hole in the aluminium shield. A close-up efficiency map with subpixel resolution covering $5 \times 2$ pixels in the transition region between the unirradiated region (left), the ring region (middle) and the center of the irradiated region (right) is shown in Fig. 6 at two different voltages $(40 \mathrm{~V}$ and $80 \mathrm{~V})$. One can notice that the lower efficiency region is at the position furthest away from the $\mathrm{n}$ - and $\mathrm{p}$-columns: that region exhibits the minimum electric field inside the sensor and charge carriers generated at this distances from the electrodes have a larger probability of being trapped.

The source of the low efficiency is under investigation. The low efficiency region could be caused by a larger radiation damage from low energy scattered protons at the edge of the Al slit holes, a real sensor effect from the abrupt transition between the irradiated and non-irradiated region or an effect of the readout chip.

In order to investigate further the possibility of a larger fluence due to low energy scattered protons, an irradiation campaign was conducted where the same aluminium shield configuration was used to irradiate several diodes of different geometries (see Fig. 7) and perform a position resolved dosimetry using the relationship between radiation induced leakage current and fluence [13]:

$$
\Delta I_{\text {leak }}\left(\phi_{e q}\right)=\alpha V \phi_{e q},
$$

where $\Delta I_{\text {leak }}$ is the radiation induced leakage current, $\phi_{e q}$ is the fluence, $V$ the volume of the sensor and $\alpha$ is a damage constant that depends on the annealing.

After irradiation to the fluence of $3.5 \times 10^{15} \mathrm{n}_{e q} / \mathrm{cm}^{2}$, the diodes were annealed for $80 \mathrm{~min}$ at $60^{\circ} \mathrm{C}$ which corresponds to a damage constant of $\alpha=4 \times 10^{-17} \mathrm{~A} / \mathrm{cm}$. The IV-curves of all the structures were measured afterwards. Since no plateau was observed in some diodes given the high fluence, the leakage current at $400 \mathrm{~V}$ was used to estimate the fluence, where the slopes of the IV-curves were found to decrease. Results are shown in Fig. 7: no significant increase in the fluence is observed in the area under the slit edge at 0 and $4 \mathrm{~mm}$ with respect to the centre of the hole. It is interesting to note that a fluence of $\sim 10^{12}-10^{13} \mathrm{n}_{e q} / \mathrm{cm}^{2}$ was measured also under the aluminium shield. Further investigation is ongoing like charge collection studies with the already irradiated diodes.

Nevertheless, the gradient in the fluence distribution expected for AFP is smaller than in the irradiated devices studied here, and the peak fluence will be 3 orders of magnitude lower 


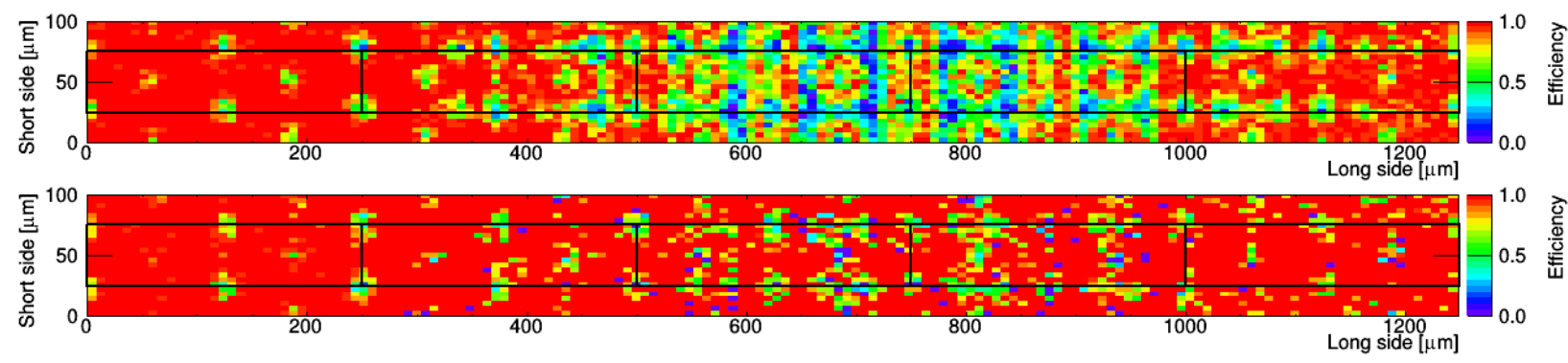

Fig. 6. Efficiency in $5 \times 2$ pixels around the transition region between non-irradiated, irradiated-ring and irradiated centre on a device irradiated at KIT with a $5 \mathrm{~mm}$ thick aluminium shield with a slit hole (CNM-S5-R7). The bias voltage was $40 \mathrm{~V}$ (top) and $80 \mathrm{~V}$ (bottom), with lower statistics. Superimposed in black lines are the position of the 6 p-columns surrounding the 2 central $n$-columns which form a pixel.
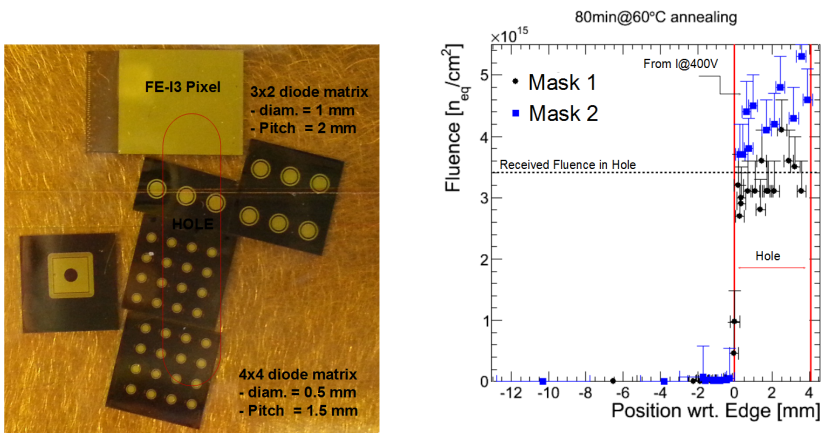

Fig. 7. Diode configuration picture (left) and fluence measurement as a function of the distance from the hole to each diode (right). The horizontal error bars represent the size of the diode, while the vertical error bars indicate the lack of plateau at the received fluence. A FE-I3 sensor was also irradiated to use the per-pixel leakage current measurement featured in the front-end, however the chip was not designed to sustain such fluences.

in the initial low-luminosity runs. Therefore, this effect is not expected to be observed in the real operation of the AFP detector.

\section{Position Resolution}

The position resolution of the tracker modules directly impacts the energy and momentum measurements of the detector and is thus critical for the physics performance of AFP. Given the non-uniform nature of the AFP irradiation profile it is also important to study the resolution for irradiated and nonirradiated areas of the tracker module, specially for the highluminosity scenario. In this section, the position resolution will be studied for a non-uniformly irradiated device, CNMS5-R7, irradiated at KIT (see Fig. 4, right) in the irradiated (under the centre of the $\mathrm{Al}$ masks hole) and non-irradiated regions separately. The following results were obtained in a testbeam at CERN SPS using the ACONITE telescope for track reconstruction, hence the pointing resolution of the configuration is $4 \pm 1 \mu \mathrm{m}$. The AFP prototype was studied with the pion beam under perpendicular incidence and biased at $80 \mathrm{~V}$.

The position resolution was measured from the difference between the position of the track in the plane of the DUT and the position of the cluster of neighbouring hits in the sensor (called residual). The cluster position is determined by a ToT weighted mean of the central position of the hits inside the cluster. The width (RMS) of the residual distribution provides a measurement of the sensor resolution convoluted with the pointing resolution of the telescope. To obtain the DUT position resolution (referred as RMS $D U T$ in Fig. 8), the telescope resolution is quadratically subtracted from the RMS of the residual distribution.

The one-directional cluster size distributions in both pixel directions are shown in Fig. 8a (non-irradiated) and 8b (irradiated). In both cases, the distributions sharply peak at 1 , as expected for a normal beam incidence. In the non-irradiated region, cluster size 2 in the short (long) pixel direction is present in $18.5 \%(2.2 \%)$ of the events. The frequency in the irradiated area, however, is slightly lower $(13.3 \%$ and $1.8 \%$ respectively). This is to be expected, since after irradiation the charge sharing decreases, diminishing the probability of 2-hit clusters in perpendicular incidence. Larger cluster sizes represent $1.8 \%(0.1 \%)$ and $1.3 \%(0.1 \%)$ of the clusters along the short (long) pixel direction in the non-irradiated and irradiated areas, respectively. The source of clusters larger than 2 are delta-electrons in the case of perpendicular incidence.

The residual distributions for different one-directional cluster sizes are shown in Fig. 8c (non-irradiated) and 8d (irradiated). The residuals of 1-hit clusters show a uniform distribution with an RMS slightly lower than the binary resolution (pitch $/ \sqrt{12}$ ). This is because the effective pixel size is smaller when restricting to cluster size 1 , since particles passing around the edge of the pixel will result in 2-hit clusters due to charge sharing. Indeed, 1-hit cluster residuals in the irradiated case are slightly wider due to charge sharing reduction caused by the radiation damage. The 2-hit cluster residuals show a better RMS due to 2-hit clusters only occurring close to the edge of two pixels in normal incidence. For cluster sizes larger than 2, a large contribution of delta-electrons arises, which introduces large tails on the corresponding residuals distributions. This effect is visible in both irradiated and non-irradiated parts of the sensor. Optimized cluster centre algorithms might help to improve the resolution and will be studied in the future.

Figure 8e (8f) shows the all-hit cluster residuals along the long (short) pixel direction. The three low peaks in the long pixel direction are caused by sub-pixel low efficiency regions.

The measured DUT position resolution for the nonirradiated $\left(15.5 \times 72.6 \mu \mathrm{m}^{2}\right)$ and irradiated $\left(15.1 \times 72.8 \mu \mathrm{m}^{2}\right)$ regions are compatible at the sub-micrometer level. The ob- 


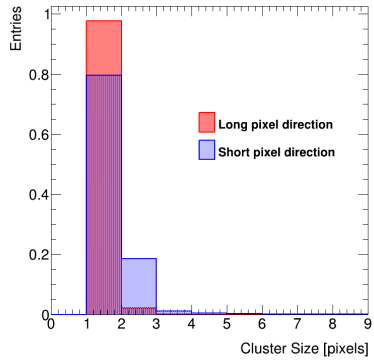

(a) Cluster size distribution in the nonirradiated region

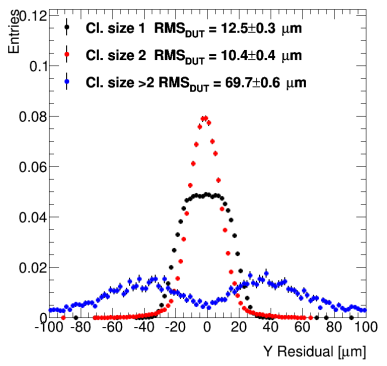

(c) Residuals in short pixel direction in the non-irradiated region

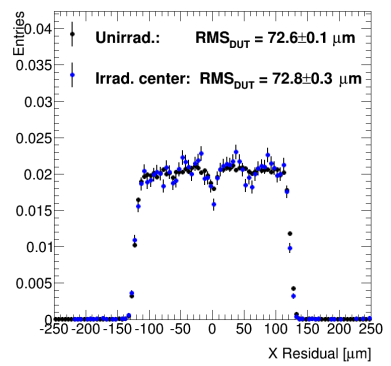

(e) Residuals in long pixel direction for (f) Residuals in short pixel direction all cluster sizes for all cluster sizes

Fig. 8. Cluster size distribution (top) and residual distribution (middle) for different cluster sizes at the non-irradiated (left) and irradiated (right) regions, and residuals for all cluster sizes (bottom) separated by irradiation area (nonirradiated and irradiated-centre), normalized to unity. The clustering of the hits was performed by ToT-weighting the central position of the fired pixels. Telescope resolution is quadratically subtracted from the RMS values to obtain the DUT resolution $\left(\mathrm{RMS}_{D U T}\right)$. The applied bias voltage was $80 \mathrm{~V}$.

tained resolution per plane is similar to the binary resolution. A combination of 4 pixel-module planes of the full tracker is expected to improve over the single plane resolution.

Further studies are envisaged regarding the position resolution at $15^{\circ}$ in future testbeams, since charge sharing induced by the small tilt is expected to improve the position resolution.

\section{CONCLUSIONS}

Characterization of FE-I4 3D sensor prototypes slim-edged for AFP has been carried out in the laboratory and in several testbeam campaigns.

Slim-edging via diamond saw technique has shown no significant degradation of the operation of the devices. The dead area reduction has been proven to be harmless to the detector down to a physical edge extension of $87-215 \mu \mathrm{m}$ in both designs (FBK and CNM). A sensitivity extension beyond the last pixel row has been observed in FBK devices, possible due to the absence of a 3D guard-ring in the FBK design. The sensitivity extension has made possible to reduce their remaining insensitive region down to less than $15 \mu \mathrm{m}$ after slim-edging before irradiation, which is only surpassed by sensors with fully active edges to date. It has been observed that this effect is more pronounced before slim-edging and it remains after irradiation, though reduced.

Non-uniform irradiation of 3D sensors has been studied in several testbeam campaigns. The irradiations were performed at a target fluence of $2-4 \times 10^{15} \mathrm{n}_{e q} / \mathrm{cm}^{2}$ (compatible with the high-luminosity AFP run scenario) at CERN-PS with a focused beam and at Karlsruhe with an Al mask with a hole to achieve a fluence gradient. High hit efficiencies were observed, of at least $96 \%$ in both irradiated and non-irradiated areas of the sensor. A small region of lower efficiency was observed around the area under the Al shield hole used in the irradiation at Karlsruhe. The possibility of higher fluence due to low energy protons was investigated by leakage current measurements of diode arrays which underwent the same irradiation as the AFP prototypes: no difference in fluence was measured around the edge of the hole. However, the expected fluence during the approved low luminosity scenario of AFP is 3 orders of magnitude lower than the studied here and in any case the gradient in the fluence distribution is expected not to be as abrupt as the one obtained with the Al mask at KIT.

The position resolution under perpendicular incidence has been measured to be around $15 \times 72 \mu \mathrm{m}^{2}$ after non-uniform irradiation at KIT, and no significant difference between irradiated and non-irradiated regions has been observed in the studied device-under-test. More studies are envisaged to investigate the resolution under a small tilt of $15^{\circ}$.

To conclude, the slim-edged 3D pixel detectors have shown their ability to fulfil the requirements of the AFP project.

\section{ACKNOWLEDGMENT}

We thank our colleagues from CNM (G. Pellegrini, M. Baselga, V. Greco) and FBK (G.-F. Dalla Betta) for providing samples and facilities and fruitful discussions. We are grateful to the AFP and RD50 collaborations for many useful hints and discussions. We thank M. Glaser (CERNPS) and F. Bögelspacher (KIT) for help with the irradiations and I. Rubinsky, A. Micelli, D. Pohl, O. Korchak and $\mathrm{Sh}$. Hsu for help during the testbeams. This work was partially funded by the MINECO, Spanish Government, under grants FPA2010-22060-C02, FPA2013-48308-C2-1-P and SEV-2012-0234 (Severo Ochoa excellence program) and the European Commission under the FP7 Research Infrastructures project AIDA, grant agreement no. 262025.

\section{REFERENCES}

[1] ATLAS Collaboration, Letter of Intent for the Phase-I Upgrade of the ATLAS Experiment, CERN-LHCC-2011-012 (2011).

[2] The ATLAS IBL collaboration, Prototype ATLAS IBL Modules using the FE-I4 Front-End Readout Chip, JINST 7 P11010 (2012). 
[3] S. Grinstein et al., Beam test studies of 3D pixel sensors irradiated nonuniformly for the ATLAS forward physics detector, Nucl. Instrum. Meth. A 699 (2013) 27.

[4] J. Lange et al., 3D silicon pixel detectors for the ATLAS Forward Physics experiment, JINST 10 (2015) C03031

[5] M. Garcia-Sciveres et al., The FE-I4 pixel readout integrated circuit, Nucl. Instrum. Meth. A 636 (2011) S155.

[6] G. Pellegrini et al., $3 D$ double sided detector fabrication at IMB-CNM, Nucl. Instrum. Meth. A 699 (2013) 27.

[7] G. Giacomini et al., Development of Double-Sided Full-Passing-Column 3D Sensors at FBK, IEEE Trans. Nucl. Sci. 60 (2013) 2357.

[8] M. Povoli et al., Design and testing of an innovative slim-edge termination for silicon radiation detectors, JINST 8 (2013) C11022
[9] C.J. Kenney, S. Parker and E. Walckiers, Results from 3-D silicon sensors with wall electrodes: near-cell-edge sensitivity measurements as a preview of active-edge sensors, IEEE Trans. Nucl. Sci. 48 (2001) 2405.

[10] O. Rohne, Edge characterization of $3 d$ silicon sensors after bumpbonding with the ATLAS pixel readout chip, IEEE Nucl. Sci. Symp. Conf. Rec. (2008) 1929.

[11] M. Dyndal, The design of the AFP detector tracking system, Presentation at: 2nd Workshop on Detectors for Forward Physics at LHC, Isola d'Elba, Italy, 29 May 2014, ATL-FWD-SLIDE-2014-231.

[12] EUDET Consortium, Detector research and development towards the International Linear Collider, Final Report (2011).

[13] M. Moll,Radiation damage in silicon particle detectors, Ph.D. Thesis, University of Hamburg, Germany (1999) DESY-THESIS-1999-040. 